Documentation et bibliothèques

DOCUMENTATION BIBLIOTHEQUES

\title{
Un nouveau service de la bibliothèque générale de l’Université d'Ottawa : la collection de brochures
}

\section{Jacques LeBlanc}

Volume 19, numéro 1, mars 1973

URI : https://id.erudit.org/iderudit/1050961ar

DOI : https://doi.org/10.7202/1050961ar

Aller au sommaire du numéro

Éditeur(s)

Association pour l'avancement des sciences et des techniques de la documentation (ASTED)

\section{ISSN}

0315-2340 (imprimé)

2291-8949 (numérique)

Découvrir la revue

\section{Citer cet article}

LeBlanc, J. (1973). Un nouveau service de la bibliothèque générale de l'Université d'Ottawa : la collection de brochures. Documentation et bibliothèques, 19(1), 34-37. https://doi.org/10.7202/1050961ar

\section{Résumé de l'article}

Les brochures constituent une masse de documents trop souvent négligés. Elles sont essentielles principalement à l'étude du XIX ${ }^{\mathrm{e}}$ siècle canadien. La bibliothèque de l'Université d'Ottawa a entrepris de constituer une collection de brochures séparée de la collection générale. L’auteur explique le traitement de cette collection spécialisée.
Tous droits réservés (C) Association pour l'avancement des sciences et des techniques de la documentation (ASTED), 1973
Ce document est protégé par la loi sur le droit d'auteur. L'utilisation des services d'Érudit (y compris la reproduction) est assujettie à sa politique d'utilisation que vous pouvez consulter en ligne.

https://apropos.erudit.org/fr/usagers/politique-dutilisation/ 


\section{Un nouveau service de la bibliothèque générale de l'Université d'Ottawa: la collection de brochures}

\author{
par Jacques LeBlanc
}

Université d'Ottawa

Les brochures constituent une masse de documents trop souvent négligés. Elles sont essentielles principalement à l'étude du XIXe siècle canadien. La bibliothèque de l'Université d'Ottawa a entrepris de constituer une collection de brochures séparée de la collection générale. L'auteur explique le traitement de cette collection spécialisée.

Le développement considérable qu'ont connu, ces dernières années, conjointement avec une renouveau certain du nationalisme, les études universitaires en civilisation canadienne et, plus spécifiquement en civilisation canadienne-française - au Québec, mais aussi en quelques centres outre-frontière, dont l'Université d'Ottawa. ${ }^{1}$ - , n'a pas manqué d'éveiller l'intérêt des chercheurs pour une source de documentation jusque-là injustement négligée, sinon méconnue, par rapport aux livres, périodiques et journaux, à savoir ce qu'on qualifie communément de "brochures".

Il faut dire d'ailleurs que les bibliothécaires doivent assumer leur part de responsabilité quant à la perpétuation de cette anomalie. Quelques-uns seulement, jusqu'à tout récemment, avaient manifesté une attention marquée pour ce genre de publication. Peu s'étaient donné la peine de combattre l'indifférence générale pour le mettre en valeur dans leur sphère d'activité, si bien qu'en plus d'un cas, la collection de brochures - dans l'hypothèse douteuse de son existence - se voyait simplement reléguée dans un local plus ou moins poussiéreux et traitée, à toutes fins utiles, en parente pauvre et insignifiante du fonds général.

Or, par obligation professionnelle, par pure curiosité d'érudit, ou encore poussé par une combinaison rare mais d'autant plus louable des deux, quiconque a tant soit peu l'occasion de consulter Dionne ou Gagnon ${ }^{2}$, pour ne mentionner que ces deux bibliographies canadiennes rétrospectives, ne peut qu'être frappé par l'ampleur de la production nationale en ce domaine. Sans doute faut-il des fréquentations livresques de ce genre pour

1 Le Centre de recherche en civilisation canadienne-francalse de l'Université d'Ottawa fut établi en 1958.

2. N.E. Dionne, Inventaire chronologique des livres... Québec, 1905-1909 (4vol.), plus un Supplément. 1912.

Philéas Gagnon Essai de bibliographie canadienne, vol

1. Québec, 1895; vol. 2, Montréal, 1913. réaliser pleinement quelle mine presque inépuisable de renseignements constituent ces opuscules, et ceci par rapport à toutes les données (sociologiques, historiques, religieuses ou autres) susceptibles d'intéresser les travailleurs intellectuels et les curieux de la petite histoire. Tant par leur quantité la production québécoise à elle seule est loin d'être recensée in extenso que par leur contenu touchant aux matières les plus variées ${ }^{3}$, et parce qu'elles reflètent souvent mieux que tout autre organe imprimé les préoccupations, les goûts ou les luttes d'une époque dont elles transmettent globalement une image extraordinairement vivante et actuel$1 e^{4}$, les brochures peuvent servir d'instrument hautement efficace à qui veut approfondir telle tranche de l'histoire, tel courant de pensée, ou encore se forger une opinion sur une question particulière, explorer le cheminement d'une crntroverse, ou tout simplement prendre le pouls d'un groupe, d'une société. Ces affirmations se vérifient en particulier pour la production canadienne du XIXe siècle, lequel ne connaissait pas encore la prolifération vertigineuse des périodiques et des livres propre à notre époque; par conséquent, la brochure jouait un rôle prépondérant dans les propagandes diverses, et servait d'exutoire aux auteurs en mal de public, ceux-là même qui, aujourd'hui, alimentent la chronique des "Lettres à l'éditeur" de nos journaux. Si le $X X e$ siècle n'a pas fait disparaître la brochure comme canal de communication pour ne pas dire comme genre littéraire, il en a davantage fait le porte-parole des bureaux d'information d'agences gouvernementales et de sociétés savantes, plus que celui des opinions personnelles.

Mais chez-nous, en des temps relativement peu lointains, il en était autrement. Plus que l'article de journal, la brochure pouvait aller en profondeur, admettant volontiers de plus amples développements et visant à une information plus étayée. Plus que le livre ou la monographie impersonnelle et froide, parce que de dimension et d'intention plus modestes et de conception plus spontanée, elle pouvait jouer le rôle de medium journalistique populaire, atteindre un vaste public, en représenter et en former l'opinion, élargissant ainsi les bases d'une controverse ou la connaissance des éléments d'un fait, d'un problème, d'une situation. Plus que lui aussi, elle apparaissait apte à couvrir un sujet d'intérêt local, une question particulière, une polémique circonscrite. Enfin, elle possédait, sur le périodique, cet avantage de n'avoir pas à se préoccuper outre mesure de la qualité du contenu, puisque l'auditoire auquel elle s'adressait se montrait plus friand d'information que de stylistique ou même, à l'occasion, d'objectivité. Ainsi, un certain type de brochure qui, à des goûts plus raffinés, serait apparu bien banal, pouvait-il jouir d'une grande vogue auprès d'une autre classe de lecteurs.

3. Voir à ce sujet: $M$. Singh, "Nature and scope of pamp hlets", Indian Librarian vol. 23, no. 3 (1968), 163 4. L Condit A pamphlet about pamphlets, Chicago, University of Chicago Press, 1939, p. 21 
Il ne s'agit donc pas ici, lorsqu'il est question de "brochures", exclusivement de "pamphlets" au sens anglais du mot tel que défini, par exemple, dans les classiques américains de la bibliothéconomie ${ }^{5}$ : courtes publications de caractère récent, habituellement gratuites et d'intérêt éphémère, de ce genre "qui meurent après avoir bourdonné un jour ou deux" ou que "vous ne lisez pas", (selon les expressions de Voltaire (d'après Littré); ainsi en serait-il, par exemple, du prospectus d'une maison de courtage. S'y assimile tout document, monographique ou non, imprimé ou non (ainsi des albums de photographies) que l'on peut physiquement ranger dans l'éventail qui va du tract à la plaquette (pouvant parfois aller jusqu'à une centaine de pages et même exceptionnellement au-delà) et qui, bibliographiquement, a été produit en relation avec une situation précise (brochure de circonstance $)^{6}$, sans prétention à une approche exhaustive du sujet, mais avec une intention polémique, descriptive, didactique ou d'information; ou bien, par contre, qui traite d'un point si particulier ou si obscur qu'un long développement ne serait ni possible, ni désirable (brochures d'érudition) ${ }^{5}$. II n'est pas facile en l'occurence d'établir une définition couvrant tous les cas, parce que la variété des thèmes, les différences de longueur et de style confondent toute généralisation hâtive. Mais on peut dire, pour éviter un certain arbitraire $^{8}$, qu'il s'agit d'un petit ouvrage ou traité (opusculum) se situant généralement en dehors de la fiction, ayant un thème cohérent de développement en relation avec un sujet intéressant immédiatement mais temporairement l'opinion publique au moment de sa parution $^{9}$; ou encore d'un ouvrage s'adressant aux préoccupations particulières d'un type spécialisé de lecteurs. Ce qui, dans son ensemble, exclut les simples feuilles de publicité ou de propagande. Disons enfin, pour écarter toute confusion, que si, par définition, tout pamphlet (court écrit satirique) est une brochure, toutes les brochures ne sont pas de nature pamphlétaire.

$$
\star \star \star \star \star \star
$$

C'est en tenant compte de ces diverses

5. Voir: M.D. Carter and W.J. Bonk, Building library collections, 3rd ed, Metuchen, N.J., Scarecrow Press, 1969, p. $100-101$.

M. F. Tauber and Associates, Technical services in libraries, New York, Columbia University Press, 1967, p. 335. 6. Les brochures publiées sur la question des écoles du Manitoba vers 1895 et celles sur les écoles françaises d'Ontario autour de 1910 illustrent bien cette catégorie. 7. Du genre: La famille d'Estimauville de Beaumouchel, de P.-G. Roy, Lévis, 1903

8. "The pamphlet is what we make it", Condit, op. cit., p. 7. "Anything you want to think of as a pamphlet", $S$. Miller, The Vertical File and its Satellites, Littleton, Colo., Libraries Unlimited, 1971, p. 67.

9. Voir les différentes définitions des encyclopédies. article "pamphlet", Chambers's Encyclopaedia, New York Arundel 1883, vol 9, p. 12. D. Bush article "pamphlet", Chambers's Encyclopaedia, New ed. London, G. Newnes, 1959, vol. 10, pp. 380-381; N. Stewart, article "pamphlet", Collier's Encyclopedia, New York PF Collier, 1959, vol. 15, p. 401; article "pamphlets". The Encyclopaedia Britannica, Edinburgh, A. and C. Black, 1885, vol. 18, p. 204; article "pamphlet", Encyclopedia International, New York, Grolier, 1964, vol. 14, p. 30 perspectives que l'Université d'Ottawa a entrepris de constituer une collection de brochures séparée de la collection générale, et traitée selon ses normes propres. Une telle politique résulte, d'abord comme il vient d'être souligné, du caractère particulier de ce genre de publication, et aussi de motifs de gérance interne. Une collection indépendante où chaque pièce est conservée dans une chemise permet d'éviter les frais de reliure (toujours onéreux) et assure à ces ouvrages qui, à cause de leur format, sont facilement subtilisés ou perdus lorsque laissés sur les rayons, une meilleure préservation, et ceci, à l'intérieur d'un système qui permet la consultation mais non la libre circulation.

Les efforts pour constituer la collection se concentrent actuellement sur les Canadiana, i.e. sur les brochures publiées au Canada depuis les origines, ou portant sur le Canada et sur des problèmes topiques lorsque publiées à l'étranger ${ }^{9 a}$. Ce cadre fixé, il n'y a théoriquement aucune limitation de sujet ${ }^{10}$; bien que l'on mette plutôt l'accent sur ce qui présente un intérêt historique. La collection accueille aussi bien le catalogue d'exposition et le prospectus de collège du XIXe siècle que les pamphlets politiques ou religieuX du XXe. Autant que possible, surtout si elles sont de caractère hétéroclite à moins qu'il ne s'agisse d'une collection ou d'un périodique les brochures reliées en volumes sont détachées et cataloguées individuellement.

Les procédures suivies se veulent simples et visent primordialement à offrir, à l'usager, un accès rapide à l'information désirée. Les brochures sont d'abord réparties par année de parution, puis, à l'intérieur de ce premier groupement, codées selon leur séquence d'accession et placées dans une chemise à étiquette correspondante, avec, en plus, le sigle de localisation départementale. Ainsi, on aura:

01- numéro du projet

$\mathrm{CRC}$ - sigle de localisation départementale (Centre de recherche en civilisation canadienne-française)

1895-1, 1895-2 etc. numérotation progressive par ordre d'entrée

le tout constituant le numéro d'identification, sans aucun autre agencement préétabli, qu'il soit alphabétique ou systématique. Les boîtes contenant les chemises sont, par la suite, disposées chronologiquement sur les rayons. Aussi, le premier problème rencontré relève$t$-il de le datation de chaque unité bibliographique. Ceux qui ont l'expérience du catalogage savent qu'il n'est pas toujours facile, voire même possible, de fixer avec certitude la date de parution à partir de la publication ellemême, les indications pertinentes faisant souvent défaut. II faut alors s'en remettre soit au contenu, soit aux ouvrages de référence, et il est rare que par ce biais, d'une façon ou d'une

9a. Ce qui inclut tout ouvrage d'auteurs canadiens 10. On y trouve ainsi quelques oeuvres littéraires ou paralittéraires, comme la traduction du "Dies irae" de P.J.O. Chauveau, Montréal, 1887, et le livre quatrième du Canada chanté d'Albert Ferland, Montréal, 1910. 
autre et avec un minimum de culture et d'intuition, l'on ne puisse établir une date à tout le moins approximative. Ce premier stage franchi, et le numéro (ou cote) d'accession donné, la brochure passe au catalogage proprement dit (établissement de la notice bibliographique), puis à l'Informathèque (détermination des vedettes-matière). Ce n'est qu'au terme de ces trois étapes que la fiche documentaire se voit complétée.

Ce catalogage, tel que pratiqué en rapport avec le projet de l'Informathèque, ne correspond qu'à une description sommaire. En un sens large, tout d'abord, parce que le numéro d'accession se substitue à la cote normale L.C. ${ }^{11}$, et, à plus strictement parler, à cause de l'omission des vedettes-matière, exception faite des biographies ${ }^{12}$. Une des caractéristiques $\mathrm{du}$ projet en question est précisément de permettre un accès au contenu par l'utilisation d'un processus de thématisation libre, i.e. d'un vocabulaire d'indexation se référant à une liste de descripteurs (thesaurus), mais aucunement de façon restrictive, donc pouvant être dépassé. Des spécialistes seront chargés par l'Informathèque de scruter chaque brochure et d'en extraire les thèmes principaux (analyse détaillée) dans une optique multidisciplinaire et sans limitation numérique ${ }^{13}$, selon le langage d'explicitation précité. Les termes d'analyse, ultérieurement codés, microfilmés puis mis en ordinateur (Miracode) constitueront autant d'éléments possibles du catalogue-index.

II s'agit, en somme, dans ses grandes lignes (recherches dans les répertoires, catalogage simplifié, regroupement, conservation, repérage), d'un système sensiblement similaire à celui déjà instauré par l'Université Laval ${ }^{14}$; ici, cependant la cote adoptée ne permet pas de préciser à quelle discipline appartient la brochure, et sous quelle forme elle existe: microfiche, microfilm ou microcarte. En conséquence, le catalogue ne comporte pas de division en sciences humaines et en sciences pures. D'autre part, pour le moment, la notice bibliographique complète n'est pas reproduite sous chaque vedette dans les entrées au catalogue (lequel il est vrai, n'est que provisoire).

Tel qu'il se présente dans son édition préliminaire de juillet 1972 , ce catalogue ${ }^{15}$ se compose de deux parties: une liste alphabétique des titres avec renvoi au code correspondant, et une liste selon l'année de parution et le numéro d'accès avec titres correspon11. L'utilisation des classifications L.C. ou Dewey apparait utile dans le cas des brochures vraiment "éphémères" et des feuilles de publicité, etc. Cf. M. Jones, "Pamphlet classification", Reference Quarterly, vol. 8 no. 1 (1968), 17 19.

12. L'exception se justifie par le fait que la datation des personnages pose souvent des problèmes et nécessite des recherches bibliographiques compliquées. De plus, il est impérieux que les dates soient uniformes malgré les sources parfois divergentes, ce qui appelle l'établissement d'un fichier-autorité.

13. La fiche d'analyse, en plus des éléments habituels (titre, date de publication, auteur, genre de publication éditeur, lieu de publication) peut recevoir 13 sujets possibles; l'on peut évidemment recourir à une fiche supplémentaire pour une brochure donnée, mais une moyenne de 8 à 12 sujets est prévue pour chaque unité.

14. Réal Gauthier, "Le service des brochures," Bulle- dants. On prévoit, pour l'édition définitive qui devrait voir le jour au cours de 1973, une triple division: liste séquentielle des codes (topographique) avec reproduction de la notice bibliographique complète; liste d'auteurs avec titres et références au numéro de code; liste par titres avec renvois similaires. Un catalogue-matières viendra plus tard couronner le tout. On espère ainsi, à l'aide de ces divers instruments, pouvoir fournir un service personnel et une orientation au niveau de la méthodologie à quiconque est engagé sérieusement dans un projet de recherche.

Une telle approche facilite évidemment, dans une certaine mesure, la tâche du catalogueur, puisqu'il ne doit se préoccupper, sur ce plan, que des entrées secondaires (personnes, collectivités, titres et numéros de collection, titres). On rencontre, malgré tout, des problèmes ardus, dont l'un des moindres n'est pas l'identification des auteurs. Les brochures anonymes ou signées d'un pseudonyme abondent, particulièrement au XIXe siècle (à preuve Audet, utile sans être exhaustif) ${ }^{16}$. Même dans les cas - les plus fréquents - où le nom de l'auteur peut être déterminé d'après la page de titre ou autrement, il ne s'avère pas toujours aisé de le situer avec exactitude dans le temps, d'autant plus qu'en de nombreuses occasions, il s'agit d'écrivains mineurs et obscurs dont le premier ouvrage a essouflé l'inspiration. L'expérience en ce domaine fait ressortir le besoin pressant d'une bio-bibliographie complète des auteurs canadiens-français en particulier, les ouvrages existants, malgré leur valeur certaine, étant nettement dépassés, insuffisants ou marginaux (Gagnon, Dionne, déjà cités, mais aussi Beauchemin, Macmillan, Tremaine et quelques autres) ${ }^{17}$. On peut espérer, à cet égard, que le Dictionnaire biographique du Canada en cours de publication ${ }^{18}$, comblera certaines de ces lacunes. Enfin, soulignons en passant que dans la plupart des cas où l'auteur fait partie du clergé séculier ou régulier, Allaire ${ }^{19}$ constitue la seule source de renseignements biographiques.

La question de l'identification et de la datation de l'auteur étant résolue, les autres étapes de la rédaction de la notice bibliographique sont en général faciles et peuvent être franchies rapidement (titre, numéro d'édition, adresse bibliographique, collation, titre et numéro de collection, notes analytiques ou autres). Une fois les processus tin de l'Unesco a l'intention des bibliothèques, vol. 24. no 3 (1970), 188-189.

15. Catalogue des pamphlets et brochures de type Canadiana. Ottawa, Université d'Ottawa, 1972.

16. F.J. Audet et G. Malchelosse, Pseudonymes canadiens, Montréal, 1936

17. J.J. Lefebvre. Le Canada, l'Amérique: géographie, histoire. Ed. rev., augm. et mise à jour. Supplèment du Dictionnaire Beauchemin canadien. Montréal, Beauche$\min , 1968$;

A Bibliography of Canadiana. Ed. by F.M. Staton and M. Tremaine. Toronto, The public Library, 1965, plus Supplement;

M. Tremaine. A Bibliography of Canadian Imprints, 1751-1800. Toronto, University of Toronto Press, 1952;

S.W. Wallace. The Macmillan Dictionary of Canadian Biography. 3rd ed. rev. and enl. Toronto, Macmillan, 1963; R.E. Watters. A Check List of Canadian Literature and Background Materials, 1628-1950. Toronto, University of 
techniques complétés - dactylographie, impression des matrices - les fiches sont insérées au catalogue principal, chacune portant la mention "Pour l'analyse détaillée, consulter l'Informathèque", ceci afin que les intéressés sachent qu'ils se trouvent en présence d'une source bibliographique traitée différemment.

Quels sont, brièvement, les avantages de ce système? Sa supériorité assumée justifiet-elle les adaptations en cause et les exceptions aux procédures habituelles regardant les livres? Nous le croyons et ce, pour plusieurs raisons qui, espérons-le, résisteront au creuset de l'expérience. L'un des principaux arguments qui s'impose à l'esprit réside sans conteste dans l'assurance, fournie par la structure même du projet, d'une meilleure préservation des entités physiques. Centres énormément fréquentés, les bibliothèques universitaires n'échappent pas, malgré les mesures de contrôle, électroniques et autres, et les vérifications plus ou moins constantes des rayons, aux appétits des collectionneurs et aux distractions des commis. Par leur format même, les brochures constituent souvent des occasions faciles d'enrichir un amateur peu scrupuleux. Pour la même raison, sur un autre plan, elles se perdent ou s'égarent aisément. En outre, souvent éditées en tirage limité, elles deviennent de ce fait précieuses avec l'âge. Physiquement protégées par leur chemise, administrativement retirées de la circulation et conservées en collection spéciale pour être confiées à un centre qui contrôle de façon beaucoup plus immédiate l'accès aux pièces de la collection, elles sont moins susceptibles d'être déplacées, échappent aux convoitises et remplissent ainsi plus assurément leur fonction de collection, en assurant la possibilité d'un meilleur usage pour la recherche. Malgré leur nature quelque peu accessoire dans le cycle de l'information, elles peuvent quand même mieux jouer leur rôle d'instrument d'une quête plus poussée dans un domaine précis.

II serait des plus intéressant d'entreprendre, par exemple, une étude globale de la production de brochures au Canada français, disons au XIXe siècle, d'en distinguer les genres $^{20}$, d'en évaluer les idéologies sous-jacentes, ou encore de cerner le caractère d'une décade à partir des opuscules auxquels elle a donné le jour. Si l'Allemagne peut s'enorgueillir de Luther, la France de JeanJacques Rousseau et de Voltaire, l'Angleterre de More, Knox, Milton, Burke, Defoe, Swift, le Canada français peut, lui aussi, se prévaloir d'auteurs de brochures et de pamphlétaires qui, sans rivaliser sur le plan de la renommée avec ces écrivains universellement reconnus, n'en possèdent pas moins des réserves de verve et d'ironie, des facilités d'éloquence et d'exposition peu communes. Arthur Buies

Toronto Press, 1960. Et on pourrait facilement prolonger la liste

18. Dictionnaire biographique du Canada, Québec, Presses de l'Université Laval. 1965

19. J.-B.-A. Allaire, Dictionnaire biographique du clergé canadien-français, Montréal, 1910-1934, (6v.)
(1840-1901), G.A. Nantel (1852-1909), Thomas Chapais (1858-1946) furent des auteurs prolifiques. L.A. Dessaulles (1819-1895), dans la controverse de la Grande guerre ecclésiastique (1873), J.M. Beausoleil (1852-1928), dans Le dernier chant des serins de Laval (1890) ont démontré, pour le genre polémique, un talent certain. Des hommes comme N.E. Dionne (1848-1917), Faucher de Saint-Maurice (1844-1897), P.G. Roy (1870-1953) se sont brillamment illustrés par des brochures à caractère historique.

Par ailleurs, quelle intéressante psychanalyse sociale ne pourrait-on pas faire à partir de la prolifération, au XIXe siècle des brochures d'affaires criminelles ${ }^{21}$ ou, au début du XXe, de celles traitant de thèmes anti-alcooliques 22 , et la sociologie religieuse, par exemple, ne saurait-elle pas trouver son profit dans l'examen de la littérature pieuse du temps pour l'étude du type de catholicisme vécu ici à la même époque? Les titres euxmêmes sont révélateurs d'une conception et d'une pratique basées sur des éléments fort marginaux ${ }^{23}$ et une recherche plus poussée ne ferait que confirmer cette impression de sclérose généralisée. De même, des problèmes nés dans le Québec du XIXe siècle les relations entre l'Eglise et l'Etat, demeurent, d'une brûlante actualité ${ }^{24}$ ou viennent à peine d'être résolus.

Nous sommes persuadé qu'on arriverait ainsi à des conclusions et des aperçus qui, sans renverser les thèses et hypothèses des historiens, pourraient néanmoins aider à nuancer, ou permettre de préciser, les idées reçues en maints milieux sur une société, ses protagonistes, ses institutions, ses moeurs, sa psychologie, ses espoirs secrets, et nous faire réaliser ce que nous savions certes depuis longtemps mais non toujours avec preuves à l'appui - qu'en tant que collectivité nos problèmes d'aujourd'hui ne sont guère que les résultantes de nos erreurs d'hier.

20. Littérature d'édification, sermons, discours poittiques, conférences, manifestes, annuaires, albums-souvenir, rapports de congrès ou de sociétés, mémoires sur des questions médicales ou agricoles, comptes rendus de célébrations, commémorations, etc.

21. Pour n'en citer que quelques-unes: Le célèbre procès d'Anaïs Toussaint convaincue de l'empoisonnement de Joseph Bisson, son mari Québec, 1857.

Procès de Pierre Barbina dit Duval pour l'empoisonnement de Julie Desilie, son épouse, Trois-Rivières, 1863. Cleophas Lachance: son crime, son procès, son exécution, Lévis, 1881

Jean Badreux, L'Affaire Demers, Montréal, 1895.

22. A titre d'exemples: R.P. Sylvain, Petit manuel antialcoolique, Rimouski. 1905.

Père Hugolin, Au fond du verre; histoires d'ivrognes, Montréal, 1908.

23. Ainsi: P.F Richaudeau, La prophétie de Blois, Québec, 1870

A.C.H. Paquet Le propagateur de la dévotion à sainte Philomène, Québec, 1880-1884.

J.E. Panneton, Un sanctuaire canadien. Montréal, 1897. $J$ Saint-Denis, Cérémonies et texte latin des prières de la bénédiction d'une cloche à l'usage du clergé. Traduites et commentées a l'usage des fidèles. Chambly, 1908

24. H. LaRue, Les corporations religieuses catholiques du Québec et les nouvelles taxes qu'on veut leur imposer. Québec, 1876.

$T$. Chapais, Les congrégations enseignantes et le brevet de capacité. $2 e$ éd. Québec. 1893. 